\section{Detectability of the deletion of a tone from a tone-plus-noise background*}

\author{
ARTHUR I. SCHULMAN \\ University of Virginia, Charlottesville, Virginia 22901
}

The deletion of a 1,000-Hz tone from a tone-plus-noise background is much harder for humans to detect than the insertion of the same tone into a background of noise. A mathematically ideal $\mathrm{O}$, oblivious to the context in which an observation interval occurs, performs equally well under conditions of insertion and deletion. Human listeners, not oblivious to context, produced the function $d_{s}=0.036 \mathrm{E} / \mathrm{N}_{\mathrm{o}}$ under deletion; the slope of this linear function is no more than half that of the typical slope of the comparable insertion function. The underlying reasons for the superiority of detection of insertion to that of deletion remain unclear.

Yes-no experiments in signal detection require listeners to distinguish intervals that contain a signal from intervals that do not. Normally a signal is an augmented stimulus like a brief tone inserted into a noisy background. But a signal may also be a diminished stimulus like a tone briefly deleted from a noisy background in which it is otherwise present. While the ability to detect "insertion" has been extensively studied (see Green \& Swets, 1966), the ability to detect "deletion" has received little attention. The present research was an attempt to determine if anecdotal evidence was correct in suggesting that the detection of a tone's deletion is more difficult than detection of its insertion.

Figure 1 schematically represents trials under both insertion (I) and deletion (D) conditions. If the ongoing intertrial stimulation is ignored, the formal equivalence of $I$ and $D$ is evident. Both I and $D$ present tone-plus-noise and noise-alone observation intervals. Under I, the tone-plus-noise event is regarded as the signal; under $\mathrm{D}$, it is the noise-alone event that assumes the role of the signal, since here the tone-plus-noise event represents no change in the ongoing stimulation. A mathematically ideal $\mathrm{O}$, oblivious to the context of this ongoing stimulation, performs equally well under $I$ and $D$; whether or not a human listener would share the ideal's indifference was the object of the present inquiry.

\section{PROCEDURE}

Listeners sat in an untreated room, each with an indicator light and six numbered response buttons before him. Observation intervals of $0.10 \mathrm{sec}$ were marked by a light

*This research was carried out while the author was a stalf member at the M.I.T, Lincoln Laboratory, a research laboratory operated with support from the U.S. Air Force. I thank R. R. Mitchell for her assistance. The findings reported here were first aired at the Sixty-eighth Meeting of the Acoustical Society of America, October 21-24, 1964 . special devices, so that the (negligible) turned off for $0.10 \mathrm{sec}$.

The tonal voltage was turned on without regard for phase and without the use of transients were determined by the response deletion. of the earphone (Permoflux PDR-8). The white noise was generated by a 6D4-tube (noise generator, Model 1390-A, Grason-Stadler). Frequencies between 100 and $7,000 \mathrm{~Hz}$ were then passed by a Spencer-Kennedy Model 302 filter and amplified. Tone and noise were mixed electrically in a resistive network and then presented over a binaural headset. The relative strength of tone to noise will be specified by the value of $E / N_{0}$, where $E$ is tonal energy for the $0.10-\mathrm{sec}$ observation interval and $\mathrm{N}_{\mathrm{o}}$ is noise power per unit bandwidth. With one exception, noted below, a desired value of $E / N_{0}$ was obtained by adjusting the tonal voltage; with this exception, the overall sound-pressure level (SPL) of the noise was maintained throughout all testing at about $65 \mathrm{~dB}$ re 0.0002 microbar. Half the trials were signal trials under both conditions, and the listeners knew this. No trial-by-trial information feedback was provided.

The listeners were 52 experimentally naive college students who were tested in small groups in a single $2 \cdot h$ session. They were paid for their services. A session consisted of nine blocks of 108 trials. Listeners rested for a few minutes between blocks. Demonstrations and 20 practice trials were provided before the first block of testing at each of the experimental conditions. Of the 52 listeners, 33 were tested under both I and D; the others were tested only under $D$. Whenever a session was devoted to both I and D, Blocks 1-3 and 7-9 were assigned to one condition and Blocks 4-6 to the other.
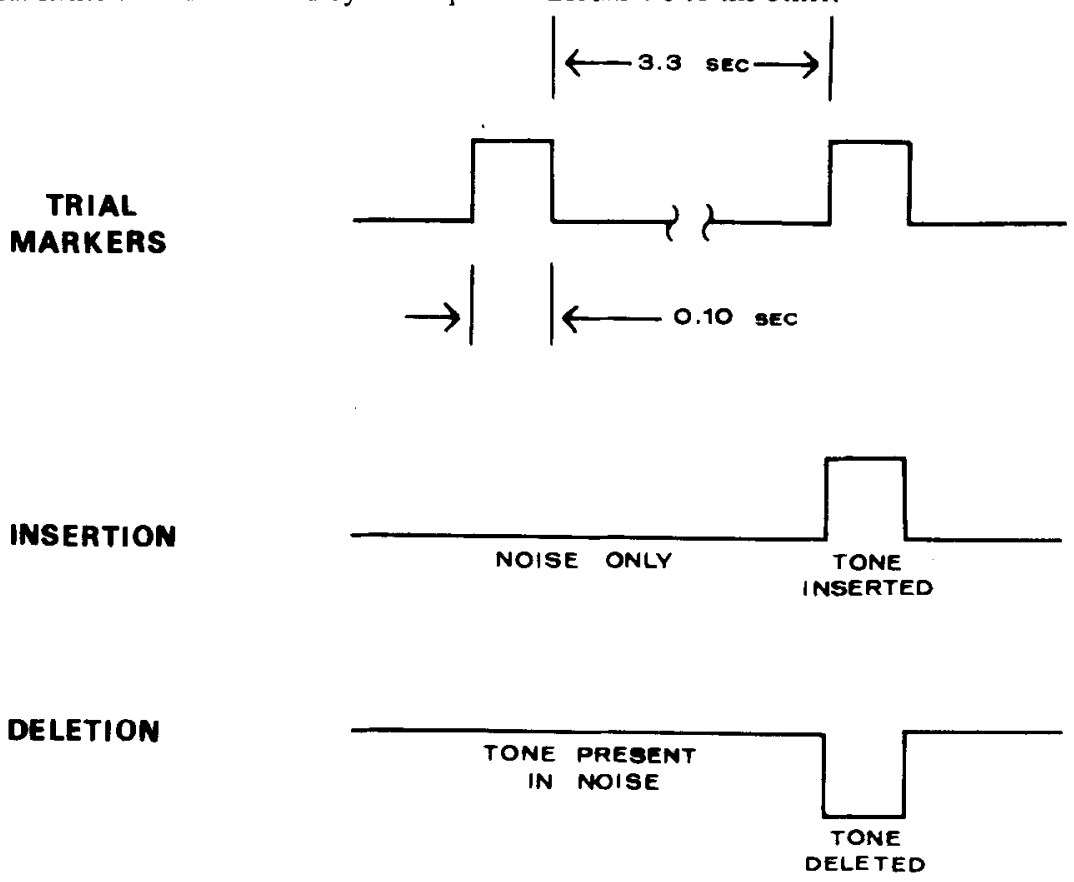

Fig. 1. Schematic illustration of tone trials and tone-plus-noise trials in insertion and 


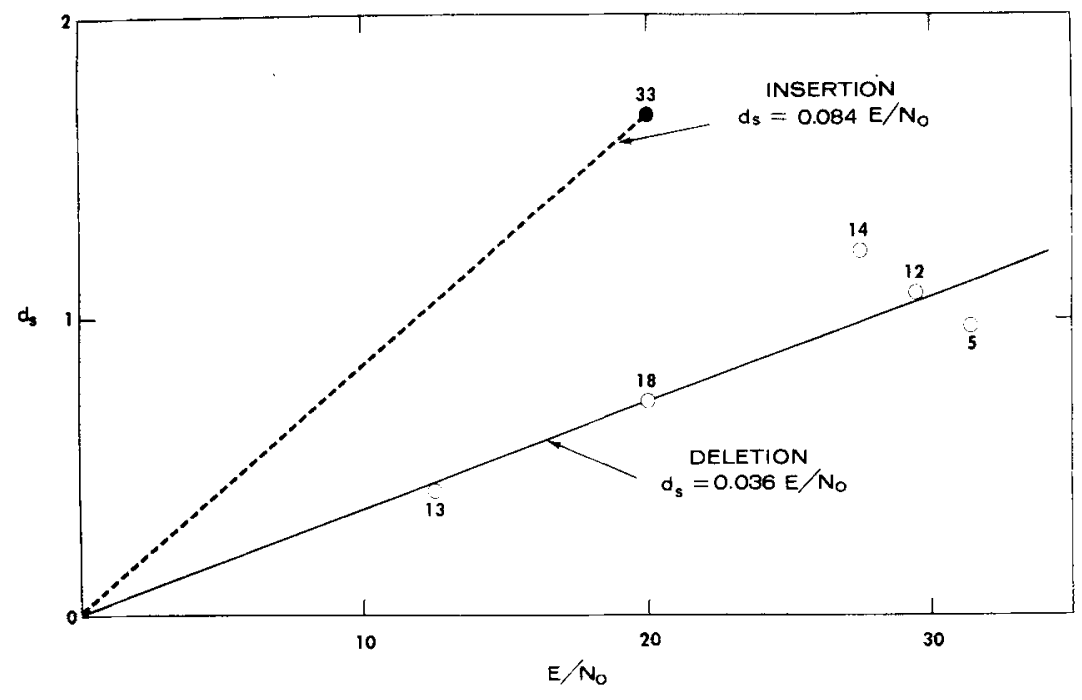

Fig. 2. Signal detectability under conditions of insertion and deletion. See text for explanation.

Listeners used a 6-point rating scale to record their confidence that a given observation interval contained a signal. In both I and D, a rating of " 1 " denoted high confidence that a signal had been presented, while a rating of " 6 " denoted high confidence that no signal had been presented. Normal use of a 6-point rating scale results in five coordinate pairs of hit and false-alarm proportions to which an operating characteristic may be fitted. A detailed description of the rating method may be found in an earlier paper (Egan, Schulman, \& Greenberg, 1959).

\section{RESULTS}

From the operating characteristic obtained for each listener under each condition, the detectability index $\mathrm{d}_{\mathrm{s}}$ was estimated. 1 Each individual $\mathrm{d}_{\mathrm{s}}$ was based on at least 324 trials. The most important results are summarized in Fig. 2 , where average $d_{s}$ is plotted against $E / N_{0}$. The figure next to each data point represents the number of listeners tested at that condition. Condition I, having been thoroughly explored in past experiments, was represented here only by $E / N_{O}=20$. In the light of earlier data (see Egan, Schulman, \& Greenberg, 1959), the dashed function of Fig. 2 seems to be a reasonable interpolation. In any event, it is clear that it is much harder to detect a tone's deletion than its insertion. In order to obtain the same value of $d_{5}$ in $D$ as in $I$, $E / N_{0}$ must be increased by more than $3.5 \mathrm{~dB}$. For the record, it should be noted that the point whose average $d_{s}$ derives from 14 listeners had its $E / N_{0}=29.5$ set by reducing the usual noise level by $1 \mathrm{~dB}$ instead of raising the level of the tone, as was the case everywhere else.

however, that overall SPL of the noise was close to $65 \mathrm{~dB}$, it becomes difficult to credit either of the above with a major role in detection. I suspect that the source of lowered detectability under $\mathrm{D}$ will be found in the nature of the running background, the degree to which it masks the tone, and in the transition from this pretrial background to a noise-alone trial. Concerning the transition, it should be noted that the same switching transients, which measurements show to be negligible, are to be found in both $I$ and $D$, although their positions at the onset and offset of the observation interval are reversed. It seems safe to ignore these transients and to consider other possible sources of disparity. Recall that $E / N_{0}$ is a measure of the relative intensity of tone to noise within the observation interval. That is to say, the intertrial stimulation is ignored in its calculation. That the intertrial stimulation matters is obvious. We know, for example, that if we turn off all sound between observation intervals and present bursts of either noise alone or of tone plus noise, detectability is depressed by $2-3 \mathrm{~dB}$, relative to normal insertion performance (see Watson, 1962, and Green, 1964). The background stimulation under $\mathrm{D}$, because of the continuous presence of the tone, was more intense than the noise-alone background under I. Consequently, the percentage increment in energy represented by a signal in I was somewhat greater than the percentage decrement in energy represented by a signal in $D$. If we think in terms of critical bands of energy, or in the width of the listener's presumed filter, these percentage increments and decrements grow further apart as the bandwidth of effective maskers is narrowed. This argument, then, leads to the prediction of better performance in I than in D. In order for a 3-dB difference to obtain, however, the effective noise voltage would have to be equal to the tonal voltage. What is more, however, is that as $\mathrm{E} / \mathrm{N}_{\mathrm{O}}$ is increased by raising the tone's voltage, the effective noise voltage would have to be raised in order to preserve the linear relationship between $d_{s}$ and $E / N_{0}$. Since in the present experiments the physical noise remains the same as $E / N_{0}$ is increased, it seems unreasonable to assume that the noise effective in masking the tone would increase to match an increase in tonal voltage.

In conclusion, it is clear that it is easier to detect tonal insertion than tonal deletion, but the reasons for insertion's superiority remain to be clarified.

\section{REFERENCES}

P., SCHULMAN, A. 1., \& determined by binary decisions and by ratings. Journal of the Acoustical Society of America, $1959,31,768-773$. EGAN, J. P., SCHENGE, Z. Operating characteristics 
GREEN, D. M. Psychoacoustics and detection theory. Journal of the Acoustical Society of America, 1960, 32, 1189-1203.

GREEN, D. M. Continuous versus gated masking for noise and sinusoidal maskers. Journal of the Acoustical Society of America, 1964, 36, 2009.

GREEN, D. M., \& SWETS, J. A. Signal detection theory and psychophysics. New York: Wiley, 1966.

GRIER, J. B. Reaction time to "tone-off." Psychonomic Science, 1966, 5, 385-386.

WATSON, C. S. Signal detection and certain physical characteristics of the stimulus during the observation interval. Doctoral dissertation, Indiana University, 1962.
NOTE

1. The index $d_{s}$ is defined as the difference between the $z$-score coordinates of the point at which the operating characteristic intersects the negative diagonal.

(Accepted for publication November 16, 1970.) 